OPEN ACCESS

Edited by:

Farhad Maleki,

McGill University, Canada

Reviewed by:

Celal Ulașoğlu,

Okan University, Turkey

Negin Forouzesh,

California State University, Los

Angeles, United States

Hamid Ceylan,

Atatürk University, Turkey

*Correspondence:

Shaohui Tang

tangshaohui206@163.com

tThese authors have contributed equally to this work

Specialty section:

This article was submitted to Computational Genomics,

a section of the journal

Frontiers in Genetics

Received: 08 September 2021 Accepted: 18 October 2021

Published: 29 October 2021

Citation:

Wu C, Zhou Y, Wang M, Dai G, Liu X, Lai $L$ and Tang $S$ (2021) Bioinformatics Analysis Explores Potential Hub Genes in Nonalcoholic Fatty Liver Disease.

Front. Genet. 12:772487.

doi: 10.3389/fgene.2021.772487

\section{Bioinformatics Analysis Explores Potential Hub Genes in Nonalcoholic Fatty Liver Disease}

\author{
Chutian Wu ${ }^{1 \dagger}$, Yun Zhou ${ }^{1,2 \dagger}$, Min Wang ${ }^{1 \dagger}$, Guolin Dai ${ }^{1}$, Xiongxiu Liu ${ }^{1}$, Leizhen Lai ${ }^{1}$ and \\ Shaohui Tang ${ }^{1 *}$ \\ ${ }^{1}$ Department of Gastroenterology, The First Affiliated Hospital, Jinan University, Guangzhou, China, ${ }^{2}$ Department of \\ Gastroenterology, The First Affiliated Hospital, Gannan Medical University, Ganzhou, China
}

Background: Nonalcoholic fatty liver disease (NAFLD) is now recognized as the most prevalent chronic liver disease worldwide. However, the dysregulated gene expression for NAFLD is still poorly understood.

Material and methods: We analyzed two public datasets (GSE48452 and GSE89632) to identify differentially expressed genes (DEGs) in NAFLD. Then, we performed a series of bioinformatics analyses to explore potential hub genes in NAFLD.

Results: This study included 26 simple steatosis (SS), 34 nonalcoholic steatohepatitis (NASH), and 13 healthy controls (HC). We observed 6 up- and 19 down-regulated genes in SS, and 13 up- and 19 down-regulated genes in NASH compared with HC. Meanwhile, the overlapping pathways between SS and NASH were PI3K-Akt signaling pathway and pathways in cancer. Then, we screened out 10 hub genes by weighted Gene CoExpression Network Analysis (WGCNA) and protein-protein interaction (PPI) networks. Eventually, we found that CYP7A1/GINS2/PDLIM3 were associated with the prognosis of hepatocellular carcinoma (HCC) in the TCGA database.

Conclusion: Although further validation is still needed, we provide useful and novel information to explore the potential candidate genes for NAFLD prognosis and therapeutic options.

Keywords: nonalcoholic fatty liver disease, nonalcoholic steatohepatitis, differentially expressed genes, hepatocellular carcinoma, bioinformatics analysis

\section{INTRODUCTION}

Nonalcoholic fatty liver disease (NAFLD) is now recognized as the most prevalent chronic liver disease worldwide, with a prevalence ranging from $13 \%$ in Africa to $42 \%$ in southeast Asia, and it may become the major cause of end-stage liver diseases by 2025 (Zarrinpar et al., 2016; Younossi, 2019; Huang et al., 2021). NAFLD represents a spectrum of disease severity, ranging from simple steatosis (SS) termed as nonalcoholic fatty liver (NAFL) to nonalcoholic steatohepatitis (NASH), cirrhosis, and hepatocellular carcinoma (HCC) (Natarajan et al., 2014). It has been well-recognized that obesity, insulin resistance, and type 2 diabetes mellitus are the strongest risk factors for NAFLD (Chen and Tian, 2020). The cause of NAFLD is multifactorial, including genetic and environmental factors (Chen and Tian, 2020). However, possible effects and underlying mechanisms for NAFLD are still not understood. Meanwhile, NAFLD-related HCC usually lacks symptoms and tends to be 
TABLE 1 | The data are shown as median (interquartile range, IQR). HC, healthy control; SS, simple steatosis; NASH, nonalcoholic steatohepatitis; BMI, body mass index; NAS, NAFLD activity score.

\begin{tabular}{|c|c|c|c|}
\hline Dataset & $\mathrm{HC}$ & SS & NASH \\
\hline GSE48452 (n) & 5 & 9 & 17 \\
\hline Gender (male: female) & $0: 5$ & $2: 7$ & $4: 13$ \\
\hline Age (years) & $45.0(35.0-62.0)$ & $37.0(32.0-46.5)$ & 47 (36-50.5) \\
\hline $\mathrm{BMI}\left(\mathrm{kg} / \mathrm{m}^{2}\right)$ & $21.0(18.8-23.5)$ & $51.9(45.7-55.7)$ & $47.8(33.4-55.7)$ \\
\hline Steatosis (\%) & $0(0-2.0)$ & $30.0(15.0-70.0)$ & 75 (70.0-85.0) \\
\hline NAS & $0.5(0-1.0)$ & $1.0(1.0-3.0)$ & $5.0(5.0-5.5)$ \\
\hline GSE89632 (n) & 8 & 17 & 17 \\
\hline Gender (male: female) & $4: 4$ & $12: 5$ & $9: 8$ \\
\hline Age (years) & $42.5(26.5-54.3)$ & $45.0(35-51.5)$ & $44.0(35.5-52.5)$ \\
\hline $\mathrm{BMI}\left(\mathrm{kg} / \mathrm{m}^{2}\right)$ & $21.2(19.9-23.1)$ & $28.9(27.5-31.3)$ & $32.0(29.65-33.6)$ \\
\hline Steatosis (\%) & $0(0-0.8)$ & $40.0(15.0-55.0)$ & $40.0(17.5-70.0)$ \\
\hline NAS & $0(0-0)$ & $2.0(1.0-2.0)$ & $5.0(4.0-6.0)$ \\
\hline
\end{tabular}

The data are shown as mean and median (interquartile range, IQR). HC, healthy control; SS, simple steatosis; NASH, nonalcoholic steatohepatitis; BMI, body mass index; NAS, NAFLD activity score.

diagnosed at a later stage and is related to poorer survival than viral hepatitis-related HCC (Younossi et al., 2015; Huang et al., 2021). In addition, NAFLD-related HCC is now proliferating and will increase in parallel with the obesity epidemic (Desai et al., 2019). Therefore, it is essential to investigate in detail the mechanism in the pathogenesis of NAFLD to find new potential targets for prognosis and therapy, especially in obese population.

Many genome-wide association studies have indicated that PNPLA3, HNF1A, NCAN, GCKR, MBOTAT, FADS1, PPAR, TNF, and TM6SF2 are important genetic and epigenetic modifiers played important roles in the pathogenesis and progression of NAFLD (Choudhary and Duseja, 2021). Meanwhile, some bioinformatics researches offer new ideas for exploring potential targets of NAFLD. Zeng et al. (2020) found that $A K R 1 B 10$ and SPP1 were related to immune cell infiltrations and associated with NAFLD progression. Liu et al. (2020) reported that TOP2A, NHP2L1, PCNA, CHEK1, ACACA, CCS, $A C A C B$ had a significant impact on NAFLD progression and were associated with HCC progression. What's more, Wang et al. (2016) indicated that Lp1, Ces2, Fasn, Hmgcs1, Sc4mol, Fads1, and Mup1 were associated with lipid metabolism, and Cbr3, Trib3, $\mathrm{Nfe} 212$ were related to oxidative stress in NAFLD mouse model. Obviously, there is significant heterogeneity between studies in both animal and human experiments. Although many studies have been devoted to exploring the pathogenesis and progression of NAFLD, there are still no effective drugs for the treatment of NAFLD except for lifestyle changes (Leoni et al., 2018). Thus, combination bioinformatics analysis with public microarray data will contribute to explore novel pathways and genes regulating NAFLD.

Therefore, we analyzed two public datasets to identify differentially expressed genes (DEGs) among healthy controls (HC), SS, and NASH. Then, Weighted Gene Co-Expression Network Analysis (WGCNA) and protein-protein interaction (PPI) networks were performed to explore the impact of DEGs on NAFLD. This study aimed to screen potential genes for NAFLD development.

\section{MATERIAL AND METHODS}

\section{Data Retrieving and Processing}

The gene expression profiles of GSE48452 (Ahrens et al., 2013) and GSE89632 (Arendt et al., 2015) were downloaded from Gene Expression Omnibus (GEO, http://www.ncbi.nlm.nih.gov/geo). To prevent the effects of overweight in the evaluation, healthy obesity with body mass index (BMI) over $24 \mathrm{~kg} / \mathrm{m}^{2}$ were excluded from the HC group. Besides, due to NAFLD commonly happened to the obese population, NALFD patients with BMI less than $24 \mathrm{~kg} / \mathrm{m}^{2}$ were also excluded from the experimental group. What is more, individuals with bariatric surgery or severely missing data at baseline were also ruled out. Finally, 9 SS samples, 17 NASH samples, and $5 \mathrm{HC}$ samples in the GSE48452, and 17 SS samples, $17 \mathrm{NASH}$ samples, and $8 \mathrm{HC}$ samples in the GSE89632 were included in this study (Table 1). HCC data were obtained from The Cancer Genome Atlas (TCGA) database, including 374 HCC samples and 50 normal samples.

For the analysis of DEGs, we used the GEO2R (https://www. ncbi.nlm.nih.gov/geo/geo2r/) to generate the R script, which used two $R$ packages (GEOquery and limma). The threshold for the DEGs was set as $p$-value $<0.05$ and $\mid \log _{2}$ fold change (FC) $\mid \geq 1$. Heat maps were drawn using $\mathrm{R}$ package "pheatmap". Venn diagram was performed using the jvenn tool (http://jvenn. toulouse.inra.fr/app/example.html), and the overlaps represented the intersection between the two datasets. Figure 1 illustrated the overall research design.

\section{Diagnostic Methods of Different States of NAFLD}

All the samples in GSE48452 and GSE89632 were validated using histological examination by a board-certified pathologist before molecular analysis, and hematoxylin and eosin (H\&E) and chromotrope aniline blue $(\mathrm{CAB})$ stained sections were used for histological analysis. The different states of NAFLD were diagnosed using criteria from NAFLD Activity Score (NAS) (Kleiner et al., 2005). 


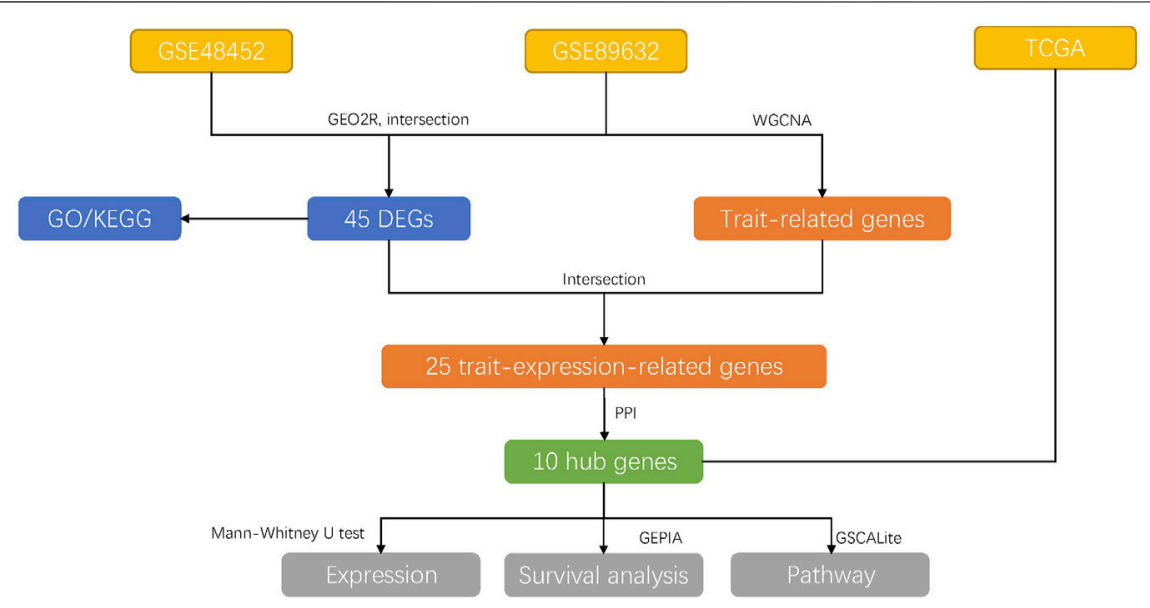

FIGURE 1 | The overall research designs. The data were downloaded from GEO and TCGA databases. Then, the DEGs were explored among the groups using GEO2R and were performed GO and KEGG analysis later, respectively. Subsequently, the GSE89632 dataset was used for WGCNA analysis to explore the trait-related genes, which were intersected with the DEGs to find trait-expression-related genes. PPI network analysis was performed to detect hub genes. Then, the expression, survival rate, and pathway of 10 hub genes were explored in the TCGA database.

\section{Gene Ontology Analysis and Kyoto Encyclopedia of Genes and Genomes Pathway Enrichment Analysis}

GO is a commonly used bioinformatics tool that supply comprehensive information on gene function of individual genomic products based on defined features and is primarily divided into three parts, molecular function (MF), biological process (BP), and cellular component (CC). KEGG is a database resource for understanding high-level biological functions and utilities. To identify the function of DEGs, GO and KEGG analysis were performed using Metascape (metascape. org) database with default settings. We determined that results were statistically significant at a level of less than 0.05 using a $p$-value. Then, histograms and bubble plots were generated with $\mathrm{R}$ package "ggplot2".

\section{Weighted Gene Co-Expression Network Analysis}

WGCNA is a well-established method for studying biological networks and diseases (Rasmussen et al., 2020). Considering that GSE89632 had more comprehensive and complete data, we used GSE89632 to detect modules highly correlated with NAFLD, and WGCNA was performed using R package "WGCNA" and carried out on all genes. The scale-free topology of the networks was assessed for various values of the $\beta$ shrinkage parameter, and we chose $\beta=5$ based on scale-free topology criterion. Finally, the dynamic tree cut algorithm was applied to the dendrogram for module identification with the mini-size of module gene numbers set as 50, and similar modules were merged following a height cutoff of 0.05 . In the module-trait analysis, gene-trait significance (GS) value $>0.3$ and module membership (MM) value $>0.55$ were defined as a threshold (Zeng et al., 2020).

\section{Protein-Protein Interaction Network Construction}

Metascape (metascape.org) database was used to construct a protein-protein interaction (PPI) network with default settings. Disconnected nodes in the network were deleted. Then, the Cytoscape software (v3.8.2) was utilized to visualize the PPI network. We used CytoHubba plugin to identify the hub genes through molecular complex detection (MCC) (Chin et al., 2014).

\section{Relationship Between Hub Gens in NAFLD and Hepatocellular Carcinoma Prognosis}

The pathway activity was acquired from GSCALite: A Web Server for Gene Set Cancer Analysis (http://bioinfo.life.hust.edu.cn/web/ GSCALite/), the survival analysis was collected from Gene Expression Profiling Interactive Analysis (GEPIA, http://gepia. cancer-pku.cn/), and the immunohistochemical pictures were collected from the Human Protein Atlas (HPA, https://www. proteinatlas.org/) database.

\section{Statistical Analysis}

Statistical analysis was performed using R software (Version 4.1.0). Statistical comparisons between groups of normalized data were performed using the t-test or Mann-Whitney U-test according to the test condition. A difference with $p<0.05$ was considered significant.

\section{RESULTS}

\section{Identification of DEGs in the NAFLD Patients}

The DEGs among HC, SS, and NASH in GSE48452 and GSE89632 datasets were identified, respectively (Figures 2A,B 
A

A

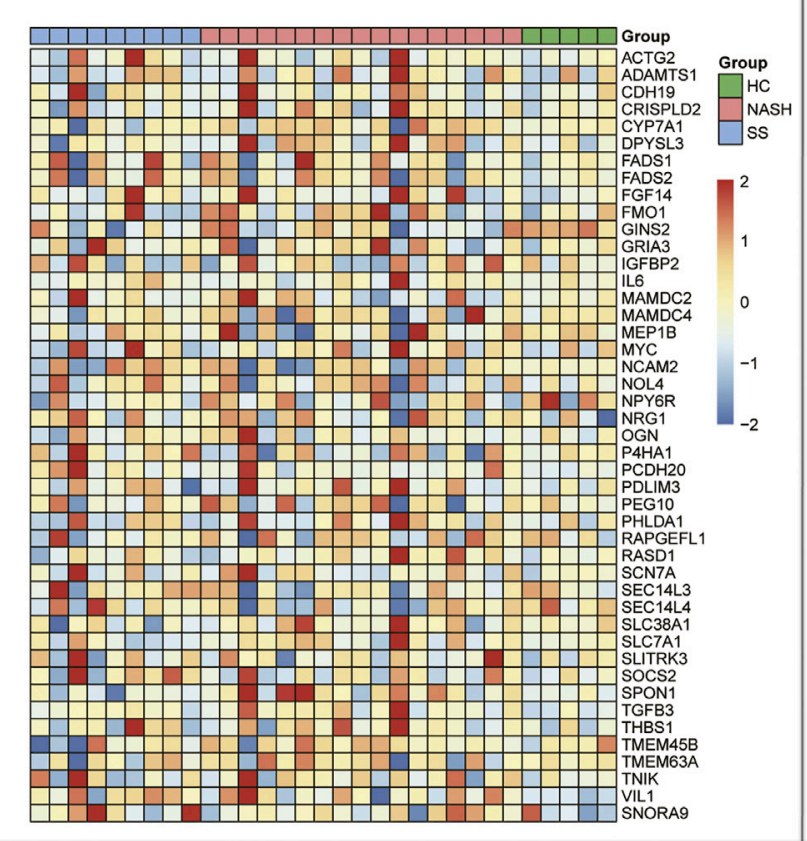

c

Up-regulated in SS vs HC

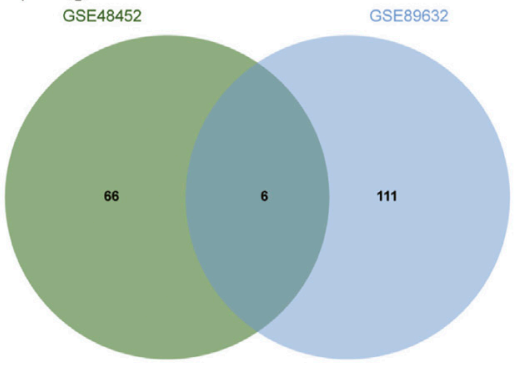

D

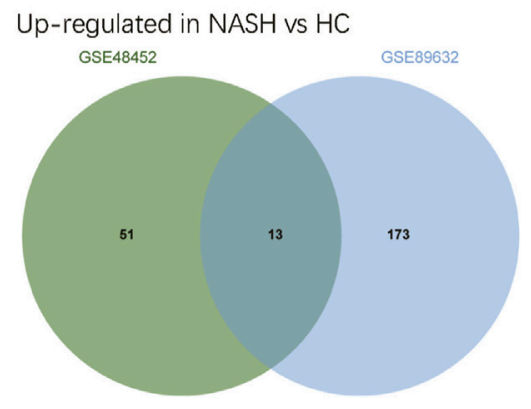

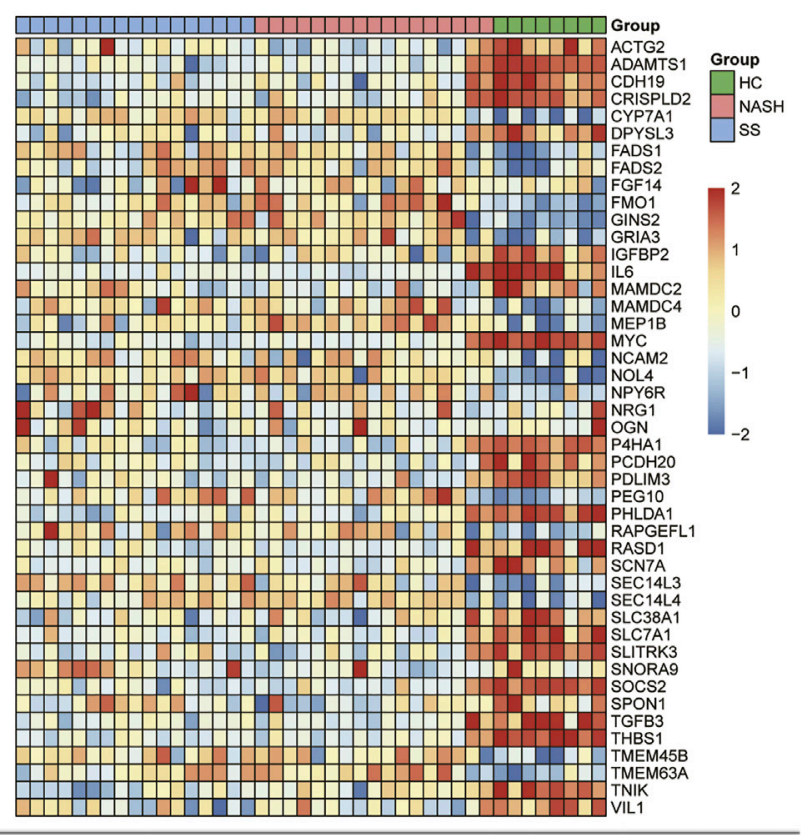

Down-regulated in SS vs $\mathrm{HC}$

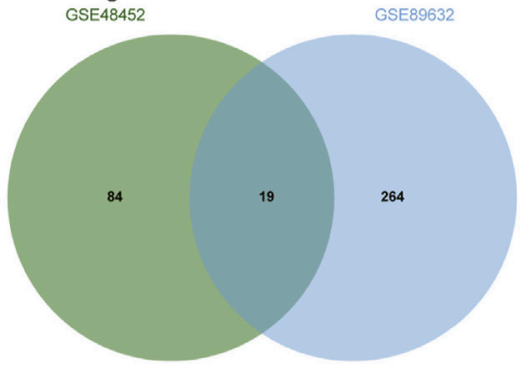

Down-regulated in NASH vs HC

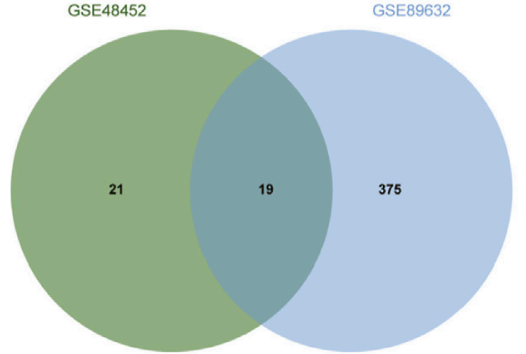

FIGURE 2 | Identification of differentially expressed genes (DEGs) among HC, SS, and NASH. (A) Heatmap of overlapping DEGs in GSE48452; (B) Heatmap of overlapping DEGs in GSE89632; (C) Venn diagrams displayed the overlapping DEGs of up- and down-regulated genes between HC and SS; (D) Venn diagrams displayed the overlapping DEGs of up- and down-regulated genes between $\mathrm{HC}$ and NASH.

and Supplementary Tables S1-S2). Then, we sought for the overlapping DEGs between the two datasets. We observed 6 upand 19 down-regulated genes in SS compared with HC (Figure 2C). We also found 13 up- and 19 down-regulated genes in NASH compared with HC (Figure 2D).

\section{GO and KEGG Pathway Enrichment Analysis}

To explore the potential roles of DEGs among HC, SS, and NASH, GO and KEGG pathway enrichment analysis were performed. The up-regulated genes between $\mathrm{HC}$ and SS were 
A
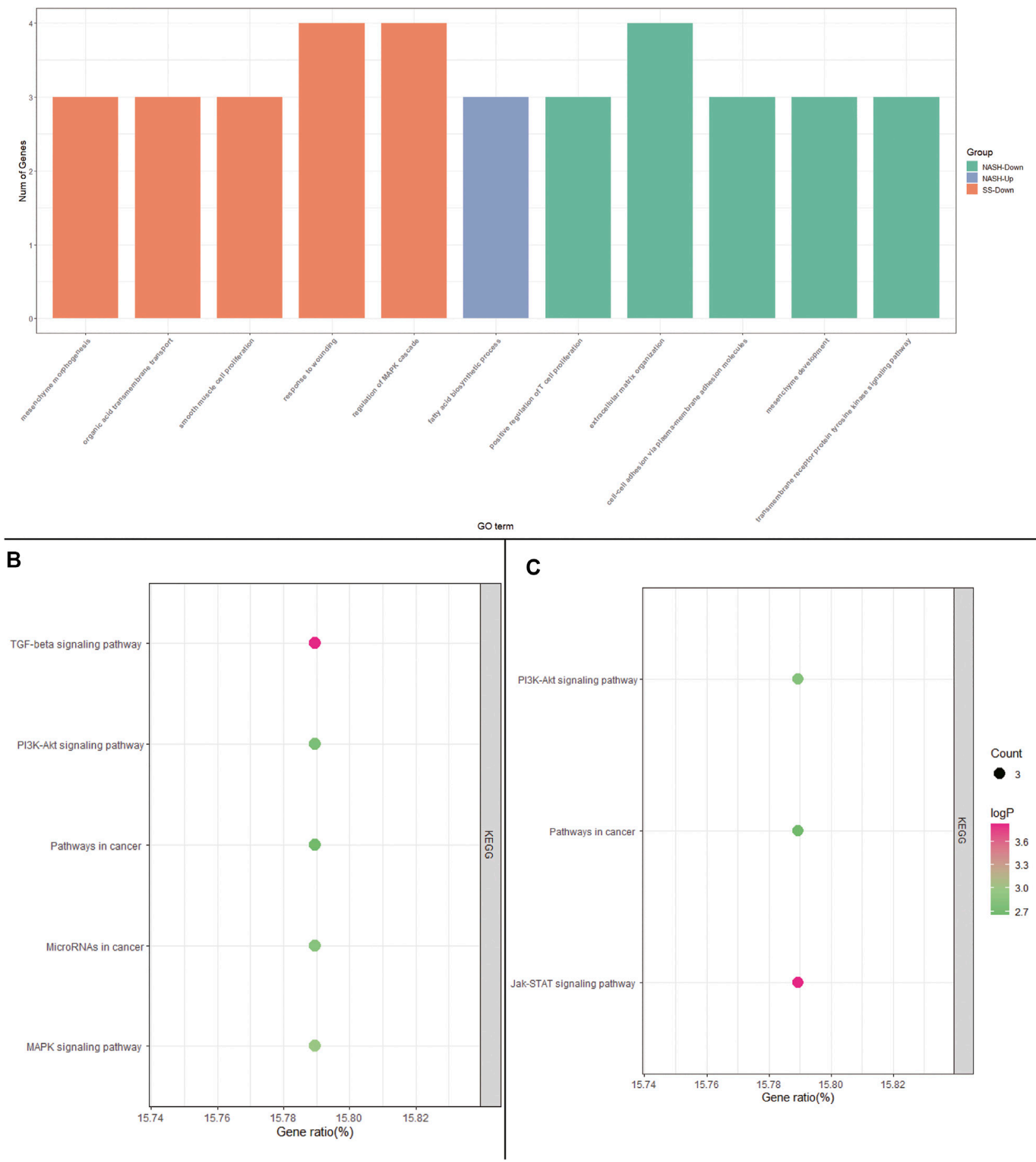

FIGURE 3 | GO and KEGG pathway enrichment analysis. (A) GO analysis of DEGs among HC, SS, and NASH; (B) KEGG analysis of down-regulated DEGs between $\mathrm{HC}$ and SS; (C) KEGG analysis of down-regulated DEGs between $\mathrm{HC}$ and NASH.

too few to allow identification of GO and KEGG pathway enrichment analysis, and the up-regulated genes between $\mathrm{HC}$ and NASH failed to enrich pathway in KEGG.
$\mathrm{GO}$ analysis showed that the down-regulated genes between $\mathrm{HC}$ and SS were mainly involved in biological processes (BP) associated with the mesenchyme morphogenesis, organic acid 


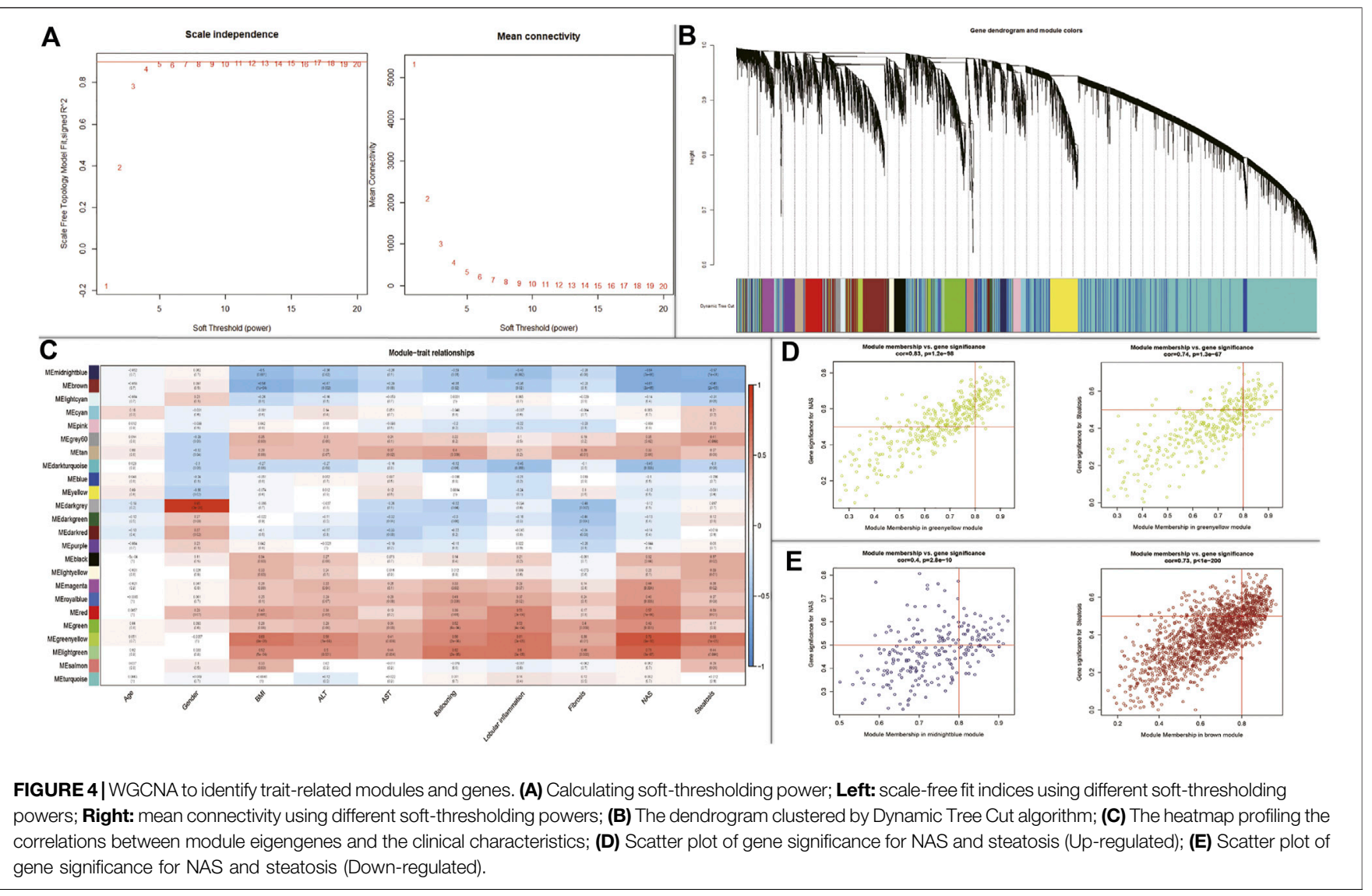

transmembrane transport, smooth muscle cell proliferation, response to wounding, and regulation of MAPK cascade (Figure 3A and Supplementary Table S3). KEGG analysis indicated that the down-regulated genes between $\mathrm{HC}$ and SS primarily enriched in TGF-beta signaling pathway, MAPK signaling pathway, MicroRNAs in cancer, PI3K-Akt signaling pathway, and pathways in cancer (Figure 3B and Supplementary Table S4).

The DEGs between HC and NASH were mainly involved in biological processes (BP) associated with fatty acid biosynthetic process, positive regulation of $\mathrm{T}$ cell proliferation, extracellular matrix organization, cell-cell adhesion via plasma-membrane adhesion molecules, mesenchyme development, and transmembrane receptor protein tyrosine kinase signaling pathway (Figure 3C and Supplementary Table S5). KEGG analysis indicated that the DEGs between $\mathrm{HC}$ and NASH were primarily enriched in Jak-STAT signaling pathway, PI3K-Akt signaling pathway, and pathways in cancer (Figure 3D and Supplementary Table S6).

\section{Identification of Key Modules by WGCNA}

WGCNA was performed to identify key modules related to clinical traits by using GSE89632 dataset. The power of $\beta=5$ (scale-free $\mathrm{R}^{2}=0.89$ ) was selected as the soft thresholding parameter to construct a scale-free network (Figure 4A). A total of 24 modules were identified (Figure 4B). Similar module clustering was constructed by using dynamic hybrid cutting (threshold $=0.05$ ). The results in Figure $4 \mathrm{C}$ showed that the greenyellow module was the highest positive module correlated to NAFLD activity score (NAS, $\mathrm{R}^{2}=0.79, p=9 \mathrm{e}^{-10}$ ) and steatosis $\left(\mathrm{R}^{2}=0.63, p=1 \mathrm{e}^{-5}\right)$. In addition, the midnightblue module was highly negative correlated to NAS $\left(\mathrm{R}^{2}=0.64, p=\right.$ $\left.7 \mathrm{e}^{-6}\right)$, and the brown module was highly negative correlated to steatosis $\left(\mathrm{R}^{2}=0.61, p=2 \mathrm{e}^{-5}\right)$. Figures $4 \mathrm{D}, \mathrm{E}$ showed the positive and negative modules.

In the module-trait analysis, we intersected the trait-related genes highly associated with NAS and steatosis and 45 DEGs generated from expression difference analysis, and finally extracted 25 trait-expression-related genes for the following analysis (Supplementary Table S7-S8).

\section{Identification of Hub Genes and Construction of Protein-Protein Interaction Network}

Subsequently, we construct a PPI network with 25 traitexpression-related genes in the Metascape database. Then, 15 filtered genes were identified (Figure 5A) and later imported into CytoHubba plugin to explore the hub genes by "MCC" methods. The results showed that MYC, TGFB3, ADAMTS1, THBS1, RASD1, PCDH20 (Down-regulated genes), MAMDC4, CYP7A1, GINS2, and PDLIM3 (Up-regulated genes) were the top 10 hub genes (Figure 5B). 

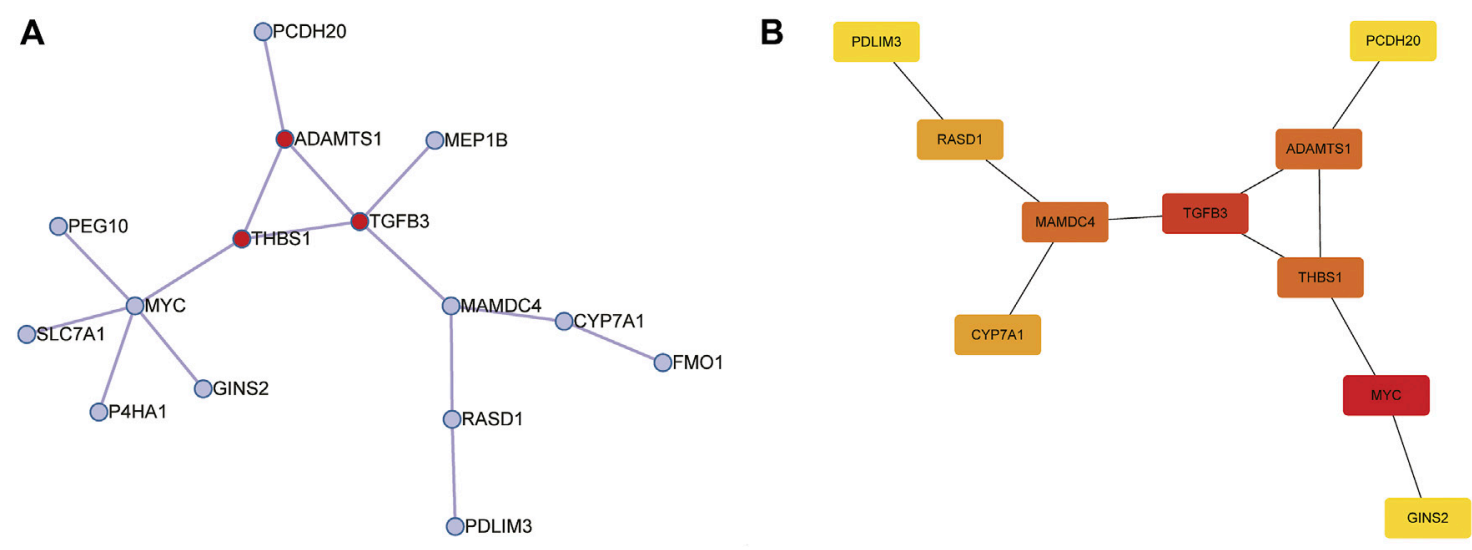

FIGURE 5 | Construction of protein-protein interaction (PPI) networks for 25 trait-expression-related genes in the Metascape database. (A) PPI network of 15 traitexpression-related genes. Red node represented the Molecular Complex Detection (MCODE) algorithm applied to identify densely connected network components. (B) Results of the CytoHubba plugin; the color changed from yellow to red was indicative of the rank of protein, and the deeper the red staining, the higher rank of protein was.

\section{Hub Genes in NAFLD Were Associated With Hepatocellular Carcinoma Prognosis}

Afterwards, the possible relationship between hub genes and hepatocellular carcinoma (HCC) was explored. We found that CYP7A1, GINS2, and PDLIM3 were significantly up-regulated, and MYC, MAMDC4, ADAMTS1, THBS1, and RASD1 were significantly down-regulated in HCC tumor samples compared with normal samples using the TCGA dataset (Figure 6A). Moreover, we found that the 8 genes above were enriched in tumor-related pathways, such as apoptosis, cell cycle, and epithelial-mesenchymal transition (EMT) (Figure 6B). Subsequently, we performed survival analysis in the genes above. As demonstrated in Figure 6C, CYP7A1-high (using quartile cutoff points) patients showed higher overall survival (OS) rates compared to CYP7A1-low patients but had no effects on disease free survival rate (DFS). What is more, compared to GINS2- high (using quartile cutoff points) and PDLIM3-high (using median cutoff points) patients, the OS rates were higher in low expression patients. In addition, GINS2-low patients showed a higher DFS rate compared to GINS2-high patients (Figures 6D,E). In the HPA database, the expression of CYP7A1/GINS2/ PDLIM3 was also abnormally elevated in HCC, but the immunohistochemical picture of CYP7A1 was missing. (Figure 6F).

\section{DISCUSSION}

Currently, the pathogenesis of NAFLD is still unclear, and the therapeutic treatments are also limited. In the present study, we identified 45 intersected DEGs between HC-SS group and HC$\mathrm{NASH}$ group, and respectively performed GO and KEGG pathway enrichment analysis to explore the potential effects of these DEGs in NAFLD. The results showed that the GO enrichments were involved in fatty acid metabolism, mesenchyme, extracellular matrix, cell adhesion, and inflammatory and immune response, which also played important roles in tumorigenesis. KEGG analysis showed that the DEGs were primarily enriched in TGF-beta signaling pathway, PI3K-Akt signaling pathway, pathways in cancer, MicroRNAs in cancer, MAPK signaling pathway, and JakSTAT signaling pathway. Both the results of GO and KEGG analysis all pointed to tumorigenesis. Meanwhile, the overlapping pathways between SS and NASH were PI3K-Akt signaling pathway and pathways in cancer, suggesting that the two pathways could be an important therapeutic target for NAFLD. The PI3K-AKT signaling pathway is known for regulating metabolism, cell growth, and cell survival. The active form of PI3K is an oncogene; thus, amplification and mutations of PI3K are usually found in many kinds of cancers (Matsuda et al., 2013). However, in this study, the PI3K-AKT signaling pathway was down-regulated in NAFLD patients. Previous studies had shown that the inhibition of PI3K-AKT signaling pathway increased hepatic insulin resistance, which exacerbated the accumulation of fat in the liver (Ntandja et al., 2020); what's more, a restoration of PI3K-AKT pathway improved the liver injury and fat accumulation ( $\mathrm{Li}$ et al., 2013). Long-duration effects of lipotoxicity aggravated the inflammatory reaction in the liver, leading to dysregulation of the PI3K-AKT signaling pathway, which might finally result in HCC (Asgharpour et al., 2016). Our findings were also consistent with previous reports (Wang et al., 2019; Liu et al., 2020).

Due to NAS and steatosis were the two main pathologic indicators in the estimation of NAFLD, we tried to find out the DEGs related to the NAS and steatosis. We identified 25 DEGs related to the NAS and steatosis, and PPI network analysis was performed to explore the hub genes in the pathogenesis and progression of NAFLD. Eventually, we determined 10 hub genes (Down-regulated genes: MYC, TGFB3, ADAMTS1, THBS1, RASD1, PCDH20; Up-regulated genes: MAMDC4, CYP7A1, GINS2, and PDLIM3) related to NAS and steatosis. 


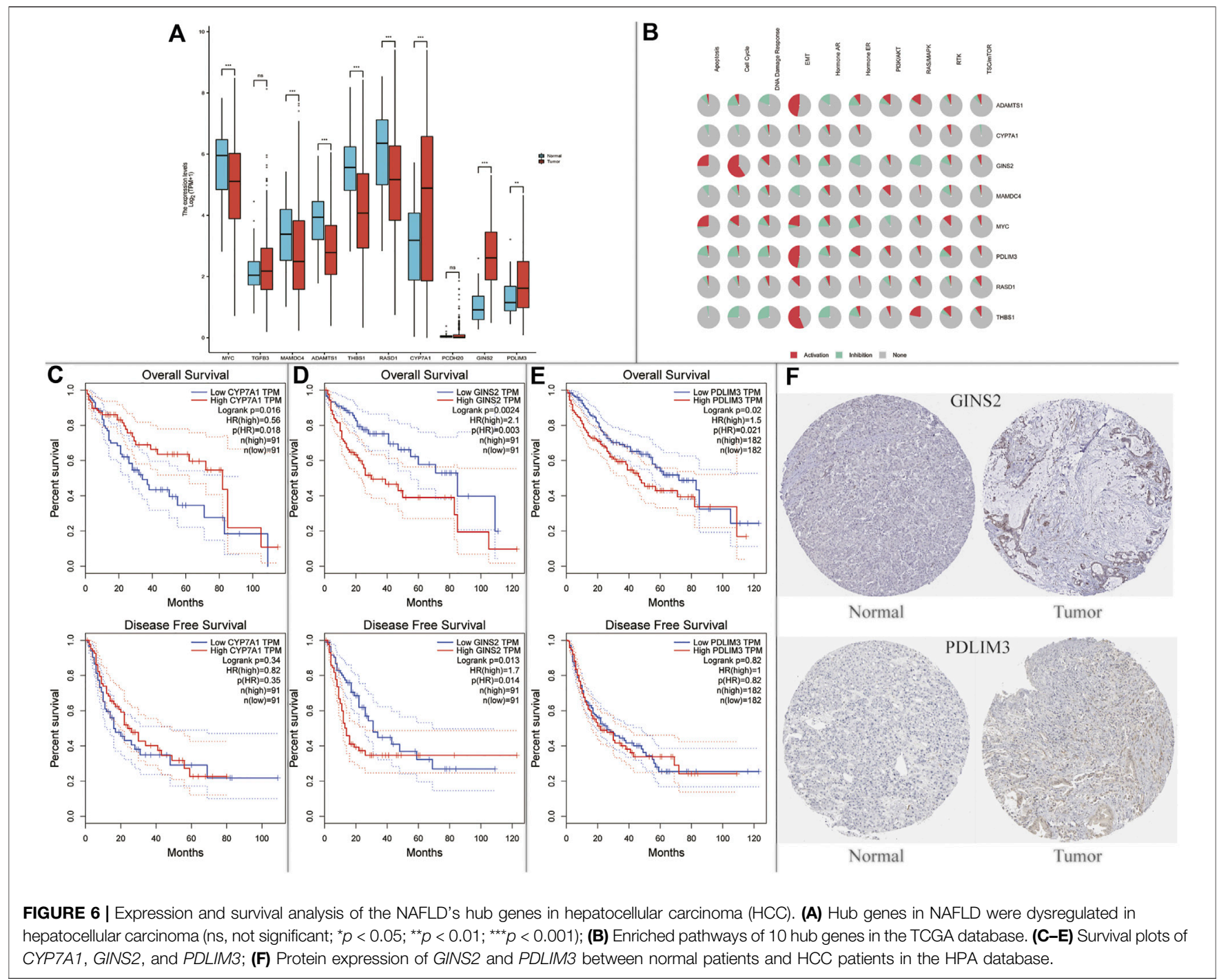

HCC is the fourth-leading cause of cancer death worldwide, and the morbidity of NAFLD-related HCC is predicted to increase dramatically by 2030 , with increases of 82,117 , and $122 \%$ from 2016 in China, France, and the USA, respectively (Yang et al., 2019; Huang et al., 2021). Therefore, we explore whether these ten hub genes were associated with the progression in HCC in the TCGA database. We found that CYP7A1, GINS2, and PDLIM3 were significantly up-regulated, and $M Y C, M A M D C 4$, ADAMTS1, THBS1, and RASD1 were significantly downregulated in HCC tumor samples compared to normal samples. Surprisingly, we also found that CYP7A1/GINS2/ PDLIM3 were correlated with HCC prognosis.

CYP7A1, catalyzing the first and rate-limiting step in the classic bile acid synthesis pathway, has been shown to be involved in lipid metabolism (Wang et al., 2020). Deficiency of CYP7A1 caused by homozygous deletion mutations can inhibit the production of bile acids, leading to the accumulation of cholesterol in the liver, reducing LDL receptors and elevating LDL cholesterol (Pullinger et al., 2002). However, CYP7A1 was up-regulated in SS and NASH group compared with HC group in our study. Previous studies have shown that CYP7A1 and its associated cholesterol processes were adversely regulated in NAFLD (Wruck and Adjaye, 2017), and glucose stimulates CYP7A1 transcription in human hepatocytes (Chiang and Ferrell, 2020). Therefore, up-regulating CYP7A1 in NAFLD may be the consequence rather than the cause of disease (Jia and Zhai, 2019). In addition, increased CYP7A1 expression and bile acid synthesis ameliorated hepatic inflammation and fibrosis, proving its anti-tumor effects (Liu et al., 2016).

GINS2, a member of the GINS family, plays a crucial role in DNA duplication and is highly expressed in various types of cancer (Kubota et al., 2003; Tian et al., 2020). However, very little research can be found about GINS2 in the liver, especially in NAFLD. Previous bioinformatics studies indicated that GINS2 might be the hub genes in the development of NASH to HCC and predicted poor prognosis in HCC, but there was no further experiment to verify its effects on NAFLD (Lian et al., 2018; Zhang et al., 2020). 
PDLIM3, highly expressed in skeletal and cardiac muscle, has been suggested to play a pivotal role in myocyte stability, signal transduction, and mechanical signaling, especially in growth and remodeling processes (Zheng et al., 2010). Interestingly, PDLIM3 was firstly screened out for a new hub gene in the pathogenesis of NAFLD and was associated with the prognosis of HCC. PDLIM3 was highly related to EMT in the GSCALite database, which might partially reveal its effects in the pathogenesis in NAFLD and HCC. More future studies are needed to gain more insights about PDLIM3.

In the present study, more attention was applied to the pathogenesis of NAFLD in obesity, which was rare in other studies. However, the present study had several limitations. Firstly, further experiments were required to verify these results. Secondly, it was hard to identify HCC patients caused by NAFLD in the TCGA database, which might impact the outcomes.

In conclusion, we analyzed two public datasets to identify DEGs among HC, SS and NASH. GO and KEGG pathway analysis revealed that the pathogenesis and progression of NAFLD were highly associated with tumorigenesis. Finally, we screened out 10 hub genes related to NAS and steatosis, and three of them were correlated with HCC prognosis. Although further validation is still needed, we provide useful and novel information to explore the potential candidate genes for NAFLD prognosis and therapeutic options.

\section{REFERENCES}

Ahrens, M., Ammerpohl, O., von Schönfels, W., Kolarova, J., Bens, S., Itzel, T., et al. (2013). DNA Methylation Analysis in Nonalcoholic Fatty Liver Disease Suggests Distinct Disease-specific and Remodeling Signatures after Bariatric Surgery. Cel Metab. 18 (2), 296-302. doi:10.1016/j.cmet.2013.07.004

Arendt, B. M., Comelli, E. M., Ma, D. W. L., Lou, W., Teterina, A., Kim, T., et al. (2015). Altered Hepatic Gene Expression in Nonalcoholic Fatty Liver Disease Is Associated with Lower Hepatic N-3 and N-6 Polyunsaturated Fatty Acids. Hepatology 61 (5), 1565-1578. doi:10.1002/hep.27695

Asgharpour, A., Cazanave, S. C., Pacana, T., Seneshaw, M., Vincent, R., Banini, B. A., et al. (2016). A Diet-Induced Animal Model of Non-alcoholic Fatty Liver Disease and Hepatocellular Cancer. J. Hepatol. 65 (3), 579-588. doi:10.1016/ j.jhep.2016.05.005

Chen, Y., and Tian, Z. (2020). Roles of Hepatic Innate and Innate-like Lymphocytes in Nonalcoholic Steatohepatitis. Front. Immunol. 11, 1500. doi:10.3389/fimmu.2020.01500

Chiang, J. Y. L., and Ferrell, J. M. (2020). Up to Date on Cholesterol 7 AlphaHydroxylase (CYP7A1) in Bile Acid Synthesis. Liver Res. 4 (2), 47-63. doi:10.1016/j.livres.2020.05.001

Chin, C.-H., Chen, S.-H., Wu, H.-H., Ho, C.-W., Ko, M.-T., and Lin, C.-Y. (2014). cytoHubba: Identifying Hub Objects and Sub-networks from Complex Interactome. BMC Syst. Biol. 8 (Suppl. 4), S11. doi:10.1186/1752-0509-8-S4-S11

Choudhary, N. S., and Duseja, A. (2021). Genetic and Epigenetic Disease Modifiers: Non-alcoholic Fatty Liver Disease (NAFLD) and Alcoholic Liver Disease (ALD). Transl Gastroenterol. Hepatol. 6, 2. doi:10.21037/tgh.2019.09.06

Desai, A., Sandhu, S., Lai, J.-P., and Sandhu, D. S. (2019). Hepatocellular Carcinoma in Non-cirrhotic Liver: A Comprehensive Review. Wjh 11 (1), 1-18. doi:10.4254/wjh.v11.i1.1

Huang, D. Q., El-Serag, H. B., and Loomba, R. (2021). Global Epidemiology of NAFLD-Related HCC: Trends, Predictions, Risk Factors and Prevention. Nat. Rev. Gastroenterol. Hepatol. 18 (4), 223-238. doi:10.1038/s41575-020-00381-6

\section{DATA AVAILABILITY STATEMENT}

The datasets presented in this study can be found in online repositories. The names of the repository/repositories and accession number(s) can be found in the article/ Supplementary Material.

\section{AUTHOR CONTRIBUTIONS}

$\mathrm{CW}, \mathrm{YZ}$, and $\mathrm{MW}$ contributed equally to this paper. $\mathrm{CW}, \mathrm{YZ}$, and MW analyzed the study data, helped draft the manuscript, made critical revisions of the manuscript. GD, XL and LL assisted with data collection and the analysis. ST supervised the research and edited the manuscript. All authors contributed to the article and approved the submitted version.

\section{ACKNOWLEDGMENTS}

The authors appreciate study investigators and staff who participated in this study.

\section{SUPPLEMENTARY MATERIAL}

The Supplementary Material for this article can be found online at: https://www.frontiersin.org/articles/10.3389/fgene.2021.772487/ full\#supplementary-material

Jia, X., and Zhai, T. (2019). Integrated Analysis of Multiple Microarray Studies to Identify Novel Gene Signatures in Non-alcoholic Fatty Liver Disease. Front. Endocrinol. 10, 599. doi:10.3389/fendo.2019.00599

Kleiner, D. E., Brunt, E. M., Van Natta, M., Behling, C., Contos, M. J., Cummings, O. W., et al. (2005). Design and Validation of a Histological Scoring System for Nonalcoholic Fatty Liver Disease. Hepatology 41 (6), 1313-1321. doi:10.1002/ hep.20701

Kubota, Y., Takase, Y., Komori, Y., Hashimoto, Y., Arata, T., Kamimura, Y., et al. (2003). A Novel Ring-like Complex of Xenopus Proteins Essential for the Initiation of DNA Replication. Genes Dev. 17 (9), 1141-1152. doi:10.1101/ gad. 1070003

Leoni, S., Tovoli, F., Napoli, L., Serio, I., Ferri, S., and Bolondi, L. (2018). Current Guidelines for the Management of Non-alcoholic Fatty Liver Disease: A Systematic Review with Comparative Analysis. Wjg 24 (30), 3361-3373. doi:10.3748/wjg.v24.i30.3361

Li, Y., Hai, J., Li, L., Chen, X., Peng, H., Cao, M., et al. (2013). Administration of Ghrelin Improves Inflammation, Oxidative Stress, and Apoptosis during and after Non-alcoholic Fatty Liver Disease Development. Endocrine 43 (2), 376-386. doi:10.1007/s12020-012-9761-5

Lian, Y.-F., Li, S.-S., Huang, Y.-L., Wei, H., Chen, D.-M., Wang, J.-L., et al. (2018). Up-regulated and Interrelated Expressions of GINS Subunits Predict Poor Prognosis in Hepatocellular Carcinoma. Biosci. Rep. 38 (6), BSR20181178. doi:10.1042/BSR20181178

Liu, H., Pathak, P., Boehme, S., and Chiang, J. L. (2016). Cholesterol 7aHydroxylase Protects the Liver from Inflammation and Fibrosis by Maintaining Cholesterol Homeostasis. J. Lipid Res. 57 (10), 1831-1844. doi:10.1194/jlr.M069807

Liu, J., Lin, B., Chen, Z., Deng, M., Wang, Y., Wang, J., et al. (2020). Identification of Key Pathways and Genes in Nonalcoholic Fatty Liver Disease Using Bioinformatics Analysis. aoms. 16 (2), 374-385. doi:10.5114/aoms.2020.93343

Matsuda, S., Kobayashi, M., and Kitagishi, Y. (2013). Roles for PI3K/AKT/PTEN Pathway in Cell Signaling of Nonalcoholic Fatty Liver Disease. ISRN Endocrinol. 2013, 1-7. doi:10.1155/2013/472432 
Natarajan, S. K., Ingham, S. A., Mohr, A. M., Wehrkamp, C. J., Ray, A., Roy, S., et al. (2014). Saturated Free Fatty Acids Induce Cholangiocyte Lipoapoptosis. Hepatology 60 (6), 1942-1956. doi:10.1002/hep.27175

Ntandja Wandji, L. C., Gnemmi, V., Mathurin, P., and Louvet, A. (2020). Combined Alcoholic and Non-alcoholic Steatohepatitis. JHEP Rep. 2 (3), 100101. doi:10.1016/j.jhepr.2020.100101

Pullinger, C. R., Eng, C., Salen, G., Shefer, S., Batta, A. K., Erickson, S. K., et al. (2002). Human Cholesterol 7a-Hydroxylase (CYP7A1) Deficiency Has a Hypercholesterolemic Phenotype. J. Clin. Invest. 110 (1), 109-117. doi:10.1172/JCI1538710.1172/jci0215387

Rasmussen, A. H., Kogelman, L. J. A., Kristensen, D. M., Chalmer, M. A., Olesen, J., and Hansen, T. F. (2020). Functional Gene Networks Reveal Distinct Mechanisms Segregating in Migraine Families. Brain 143 (10), 2945-2956. doi:10.1093/brain/awaa242

Tian, W., Yang, X., Yang, H., and Zhou, B. (2020). GINS2 Functions as a Key Gene in Lung Adenocarcinoma by WGCNA Co-expression Network Analysis. Ott Vol. 13, 6735-6746. doi:10.2147/OTT.S255251

Wruck, W., and Adjaye, J. (2017). Meta-analysis Reveals Up-Regulation of Cholesterol Processes in Non-alcoholic and Down-Regulation in Alcoholic Fatty Liver Disease. Wjh 9 (8), 443-454. doi:10.4254/wjh.v9.i8.443

Wang, C., Tao, Q., Wang, X., Wang, X., and Zhang, X. (2016). Impact of High-Fat Diet on Liver Genes Expression Profiles in Mice Model of Nonalcoholic Fatty Liver Disease. Environ. Toxicol. Pharmacol. 45, 52-62. doi:10.1016/ j.etap.2016.05.014

Wang, H., Liu, Y., Wang, D., Xu, Y., Dong, R., Yang, Y., et al. (2019). The Upstream Pathway of mTOR-Mediated Autophagy in Liver Diseases. Cells 8 (12), 1597. doi:10.3390/cells8121597

Wang, Y., Gunewardena, S., Li, F., Matye, D. J., Chen, C., Chao, X., et al. (2020). An FGF15/19-TFEB Regulatory Loop Controls Hepatic Cholesterol and Bile Acid Homeostasis. Nat. Commun. 11 (1), 3612. doi:10.1038/s41467-020-17363-6

Yang, J. D., Hainaut, P., Gores, G. J., Amadou, A., Plymoth, A., and Roberts, L. R. (2019). A Global View of Hepatocellular Carcinoma: Trends, Risk, Prevention and Management. Nat. Rev. Gastroenterol. Hepatol. 16 (10), 589-604. doi:10.1038/s41575-019-0186-y

Younossi, Z. M. (2019). Non-alcoholic Fatty Liver Disease - A Global Public Health Perspective. J. Hepatol. 70 (3), 531-544. doi:10.1016/j.jhep.2018.10.033
Younossi, Z. M., Otgonsuren, M., Henry, L., Venkatesan, C., Mishra, A., Erario, M., et al. (2015). Association of Nonalcoholic Fatty Liver Disease (NAFLD) with Hepatocellular Carcinoma (HCC) in the United States from 2004 to 2009. Hepatology 62 (6), 1723-1730. doi:10.1002/hep.28123

Zarrinpar, A., Gupta, S., Maurya, M. R., Subramaniam, S., and Loomba, R. (2016). Serum microRNAs Explain Discordance of Non-alcoholic Fatty Liver Disease in Monozygotic and Dizygotic Twins: a Prospective Study. Gut 65 (9), 1546-1554. doi:10.1136/gutjnl-2015-309456

Zeng, F., Zhang, Y., Han, X., Zeng, M., Gao, Y., and Weng, J. (2020). Predicting Non-alcoholic Fatty Liver Disease Progression and Immune Deregulations by Specific Gene Expression Patterns. Front. Immunol. 11, 609900. doi:10.3389/ fimmu.2020.609900

Zhang, D., Liu, J., Xie, T., Jiang, Q., Ding, L., Zhu, J., et al. (2020). Oleate AcidStimulated HMMR Expression by CEBPa Is Associated with Nonalcoholic Steatohepatitis and Hepatocellular Carcinoma. Int. J. Biol. Sci. 16 (15), 2812-2827. doi:10.7150/ijbs.49785

Zheng, M., Cheng, H., Banerjee, I., and Chen, J. (2010). ALP/Enigma PDZ-LIM Domain Proteins in the Heart. J. Mol. Cel Biol. 2 (2), 96-102. doi:10.1093/jmcb/ mjp038

Conflict of Interest: The authors declare that the research was conducted in the absence of any commercial or financial relationships that could be construed as a potential conflict of interest.

Publisher's Note: All claims expressed in this article are solely those of the authors and do not necessarily represent those of their affiliated organizations, or those of the publisher, the editors and the reviewers. Any product that may be evaluated in this article, or claim that may be made by its manufacturer, is not guaranteed or endorsed by the publisher.

Copyright (c) 2021 Wu, Zhou, Wang, Dai, Liu, Lai and Tang. This is an open-access article distributed under the terms of the Creative Commons Attribution License (CC $B Y)$. The use, distribution or reproduction in other forums is permitted, provided the original author(s) and the copyright owner(s) are credited and that the original publication in this journal is cited, in accordance with accepted academic practice. No use, distribution or reproduction is permitted which does not comply with these terms. 